\title{
Arthroscopic Glenoid Bone Augmentation Using Iliac Crest Autograft Is Safe and Effective for Anterior Shoulder Instability With Bone Loss
}

\author{
Matthew Oldfield, B.Sc., M.D., Joseph Burns, M.D., F.A.A.O.S., and \\ Ivan Wong, M.D., F.R.C.S.C., M.A.cM., Dip. Sports Med, F.A.A.N.A.
}

\begin{abstract}
Purpose: To establish a safety profile for an arthroscopic anatomic glenoid reconstruction using autologous iliac crest bone graft to treat shoulder instability with significant bone loss and to evaluate short-term clinical and radiological outcomes. Methods: A retrospective analysis of prospectively collected data was conducted for the patients who were treated for shoulder instability with bone loss using arthroscopic autologous iliac crest bone graft between November 2014 and June 2018. The safety profile was established by detective intraoperative or postoperative complications such as neurovascular injuries, infections, major bleeding, and subluxations. Short-term clinical and radiologic outcomes also were evaluated. Results: Thirteen patients were included in the study. A safety profile was observed, with no occurrence of intraoperative complications, neurovascular injuries, infection, or major bleeding. There were no dislocations or positive apprehension tests on clinical examination postoperatively. Postoperative Western Ontario Shoulder Instability (WOSI) scores were significantly greater than preoperative WOSI scores, with a mean improvement of $35.0 \pm 20.2(P<$ $.001)$. Twelve patients $(92.3 \%)$ received postoperative computed tomography scans, with 11 of 12 patients $(91.7 \%)$ displaying complete graft union. Conclusions: Arthroscopic treatment of shoulder instability with bone loss via autologous iliac crest bone graft is shown to be a safe operative procedure that results in favourable short-term clinical and radiologic outcomes, with a significant improvement in WOSI scores and high rates of graft union. Although graft resorption was seen in most patients who had postoperative computed tomography imaging, there were no instances of clinical graft failure. Level of Evidence: Level IV, therapeutic case series.
\end{abstract}

I nstability of the anterior shoulder resulting from trauma is associated with various injuries, both softtissue and osseous in nature. Bankart lesions for example, which involve detachment of the anterior fibrocartilaginous glenoid labrum, have been found in $68 \%$ of patients with recurrent shoulder dislocations. ${ }^{1}$ Examples of significant osseous defects may include engaging Hill-Sachs lesions of the humeral head or inverted-pear lesions of the anterior glenoid. These lesions are present in up to $22 \%$ of patients following an

From the Department of Surgery, Faculty of Medicine, Dalhousie University, Halifax, Nova Scotia Canada (M.O., I.W); and Department of Orthopedics and Shoulder Surgery, Wellstar Medical Group, Alpharetta, Georgia, U.S.A. (J.B.).

The authors report that they have no conflicts of interest in the authorship and publication of this article. Full ICMJE author disclosure forms are available for this article online, as supplementary material.

This study was performed at Queen Elizabeth Health Sciences Center, Halifax, Nova Scotia, Canada.

This paper has been presented at the 2019 Canadian Orthopaedic Association (COA) Annual Meeting as a podium presentation and at the 2019 initial dislocation of the shoulder and up to $88 \%$ of patients with recurrent instability. ${ }^{2}$ Furthermore, 50\% of patients with recurrent instability have been found to have significant glenoid bone loss greater than $10 \% .^{3}$

It has been observed that rates of postoperative instability recurrence, either subluxation or redislocation, are much greater when a significant bone defect is present. While soft-tissue lesions are commonly successfully repaired arthroscopically with postoperative subluxation recurrence rates reported as

\footnotetext{
International Society of Arthroscopy, Knee Surgery and Orthopaedic Sports Medicine (ISAKOS) Congress as a poster.

Received February 1, 2021; accepted July 23, 2021.

Address correspondence to Dr. Ivan Wong, M.D., F.R.C.S.C., M.A.cM., Dip. Sports Med, F.A.A.N.A., Department of Surgery, Faculty of Medicine, Dalhousie University, 5955 Veteran's Memorial Lane, Room 2106 VMB, Halifax, Nova Scotia, Canada.B3H2E1 Halifax, Canada.E-mail:iw@drivanwong.com

(C) 2021 THE AUTHORS. Published by Elsevier Inc. on behalf of the Arthroscopy Association of North America. This is an open access article under the CC BY-NC-ND license (http://creativecommons.org/licenses/by-nc-nd/4.0/). 2666-061X/21137

https://doi.org/10.1016/j.asmr.2021.07.023
} 
low as $4 \%$, the rate of instability recurrence following arthroscopic Bankart repair is $67 \%$ in patients who also have glenohumeral bone deficits present. ${ }^{4}$ Besides glenoid or humeral bone loss, other risk factors for greater failure rates following Bankart repair include being younger than 20 years of age, participation in competitive contact sports, and shoulder hyperlaxity., ${ }^{5,6}$

For patients with significant glenoid bone loss and other risk factors for failure of Bankart repair, procedures involving augmentation of the anterior glenoid with a bone graft yield lower rates of recurrent instability. ${ }^{7-9}$ These procedures include the Latarjet-Bristow technique (transfer of an autologous coracoid graft to the anterior glenoid), distal tibial allograft, and iliac crest autograft augmentation techniques. ${ }^{10}$ While the Latarjet-Bristow procedure is traditionally viewed as the gold-standard treatment in the context of recurrent anterior shoulder instability with bony defects, it is a nonanatomic procedure associated with rates of intraand postoperative complications as high as 30\%, including injury to the axillary, suprascapular, and musculocutaneous nerves. ${ }^{11}$ Repair via open iliac crest autograft also yields good clinical outcomes but shares similar risks to the Latarjet procedure. ${ }^{10}$

Both the Latarjet-Bristow and open iliac crest autograft procedures require splitting of the subscapularis muscle for their respective grafts to be positioned at the anterior glenoid, thereby increasing risk of injury to the axillary and musculocutaneous nerves. ${ }^{10,11,12}$ With issues such as this in mind, minimally invasive shoulder surgery techniques have continued to be developed and used in recent years, with arthroscopic treatment of severe instability with bone loss now possible using coracoid autograft, ${ }^{12,13}$ iliac crest autograft, ${ }^{14}$ and distal tibial allograft. ${ }^{15}$ Specifically, Wong and Urquhart ${ }^{15}$ developed and published a technique involving an allarthroscopic anatomic glenoid reconstruction with distal tibial allograft using an additional medial portal created parallel to the glenoid surface from an insideout technique. This technique allows the distal tibial allograft to be passed to the anterior glenoid without damaging the subscapularis muscle and neurovascular structures.

This aforementioned portal, referred to as the Halifax portal, ${ }^{16}$ is now also being used for an all-arthroscopic anatomic glenoid reconstruction (AAGR) with iliac crest autograft. As the graft is sourced from the patient, benefits of this technique include excellent graft availability and lower procedural costs compared with techniques using an allograft. The purposes of this study were to establish a safety profile for an AAGR using autologous iliac crest bone graft to treat shoulder instability with significant bone loss and to evaluate short-term clinical and radiologic outcomes. We hypothesized that AAGR using an autologous iliac crest bone graft would show a good safety profile and promising short-term outcomes for treating anterior shoulder instability with significant glenoid bone loss.

\section{Methods}

\section{Design}

This study was a retrospective analysis of data collected prospectively from patients who underwent arthroscopic treatment of shoulder instability using iliac crest autograft augmentation, performed by the 2 senior authors (I.W. and J.B.) between November 2014 and June 2018 in the 2 institutions in Canada and the United States, respectively. This study was approved by the Nova Scotia Health Authority Research Ethics Board.

\section{Patients}

From November 2014 to June 2018, the patients who underwent arthroscopic treatment of shoulder instability using iliac crest autograft augmentation were included in the study. This population consisted of both patients undergoing a primary surgery for treatment of anterior instability and those undergoing a revision surgery due to a previous failed procedure, such as a Bankart repair. Patients with neither a preoperative nor a postoperative Western Ontario Shoulder Instability Index (WOSI) score would be excluded from the study.

\section{Surgical Technique}

All procedures were performed arthroscopically. Patients were placed under general anesthetic and positioned in lateral decubitus. To begin, diagnostic arthroscopy was performed. The rotator interval was then excised with the conjoint and coracoacromial ligaments exposed. The medial Halifax portal was then created anteriorly via an inside-out technique as previously described by Wong and Urquhart. ${ }^{15}$ Next, an arthroscopic measurement of glenoid bone loss was taken. The anterior glenoid neck was then prepared for graft placement by radiofrequency ablation and mechanical shaving to expose and flatten its surface. With the medial Halifax portal created and the anterior glenoid prepared, the iliac crest bone graft was harvested from the patient's ipsilateral side using an osteotome and/or a microsaggital saw. To properly reconstitute lost glenoid bone, graft size depended on the degree of defect presented. A graft would generally be measured 0.5 to $1 \mathrm{~cm}$ in anterior-to-posterior dimension and 2 to $2.5 \mathrm{~cm}$ in superior-to-inferior dimension. After the graft was harvested, it was prepared on the back table and attached to a handled double-barreled arthroscopic cannula (Depuy Mitek, Inc., Raynham, MA). It was inserted into the shoulder via the Halifax portal, lateral to the conjoint tendon, superior to the subscapularis, and fixed to the anterior inferior glenoid with 2 bicortical cannulated screws as previously described by 
John and Wong. ${ }^{17}$ Following graft fixation, a Bankarttype repair was performed to repair the previously torn/ released capsulolabral tissue back to the native glenoid with 3 suture anchors. Finally, the shoulder was taken through a range of motion to ensure the humeral head was stable.

\section{Postoperative Rehabilitation}

Following their procedure, all patients were discharged home with their shoulder placed in neutral rotation via a brace for 6 weeks. Each patient was asked to engage in a physical therapy protocol consisting of passive mobilization exercises up to neutral rotation for the first 2 weeks, then increasing gradually to assisted active and active range of motion (ROM) exercises with a goal of full active ROM by 6 to 7 weeks. Once full ROM was achieved, focus was shifted towards strengthening the surrounding muscles and scapular control. A computed tomography (CT) scan would typically be conducted between 6 and 9 months following surgery to confirm bony union, after which return to sport would be permitted.

\section{Data Collection}

Once determined to meet inclusion criteria, participants' demographic information including operative side, sex, and age at time of procedure was collected from their medical charts. Other information collected from their charts included clinical characteristics such as past shoulder surgeries, the presence and extent of glenoid and humeral bone loss, and previous fractures. To accomplish our primary objective of establishing a safety profile, a chart review to assess for the occurrence of intraoperative complications such as neurovascular injury or major bleeding was performed. Occurrence of postoperative complications such as infection, subluxation, or dislocation was also assessed. The measurements of graft positioning were based on the axial plane for medial-lateral (ML) steps and the sagittal plane for the vertical graft positions, respectively. The ideal graft positions are flush with glenoid (i.e., ML step $=0$ ) and are at 3 to 5 o'clock.

The secondary objective of evaluating short-term clinical outcomes was accomplished through the comparison of preoperative and postoperative WOSI index scores. The WOSI index is a validated patient-reported outcome measure for patients with shoulder instability that assesses the impact of injury on shoulder function within domains of physical function, recreation, lifestyle and emotional well-being. ${ }^{18,19}$ All patients included in the study completed WOSI questionnaires preoperatively and at a minimum approximately 6 months postoperatively. The WOSI questionnaires were self-administered by the patients. The WOSI scores were reported out of 100 points and the minimal clinically important difference of WOSI is 10.4. ${ }^{19}$
Short-term radiologic outcomes also were evaluated via postoperative diagnostic imaging to assess for graft union and reabsorption. To accomplish this, anteroposterior, lateral, and axial radiographs were obtained routinely preoperatively, at 2 weeks postoperatively to rule out fractures and screw malpositioning, and again at approximately 1 year following surgery. Patients also underwent CT preoperatively to measure glenoid bone loss and at approximately 6 months postoperatively to assess for bone healing before being cleared for return to sport. All postoperative CT scans were also used to further assess bony union and graft resorption, as well as to ensure proper placement of the bone graft relative to the glenoid while measuring preoperative and postoperative sagittal dimensions of the glenoid. All CTs were reviewed by the senior author (I.W.).

\section{Statistical Analysis}

Statistical analysis of the data was carried out with multiple 2 -sided paired $t$ tests and one-way analysis of variance, with frequencies and percentages reported for categorical variables while means with standard deviations were reported for continuous variables. Patients with different graft resorption levels were compared using analysis of variance in terms of their postoperative anteroposterior dimensions of grafts, and glenoid with and without grafts. To estimate the effect size of the changes from preoperative to postoperative WOSI scores, Hedges' $\mathrm{g}$ was selected and calculated due to the small sample size of this study. The analysis was conducted using the SPSS (version 25, IBM Corp., Armonk, NY) with a significance level of .05.

\section{Results}

From November 2014 to June 2018, 16 patients met the inclusion criterion and 2 patients were excluded due to being without any WOSI scores, leaving a total of 14 patients to be included in the study. One patient was lost to follow-up; therefore, 13 patients with a mean follow-up of 1.4 years (range: 0.33-3.73 years) were finally included in the analysis (Fig 1). With either preoperative CT or diagnostic arthroscopy, all patients included in the study were found to have glenoid bone loss $(20 \%-45 \%)$. The demographics are summarized in Table 1.

\section{Safety Profile}

No intraoperative complications were observed in this case series such as neurovascular injury or major bleeding. There were no postoperative complications including hardware complications and consequently there was no need for revision surgeries for hardware removal. One patient was found to have partial graft nonunion; however, this was asymptomatic and the patient did not require further surgery. Three patients 

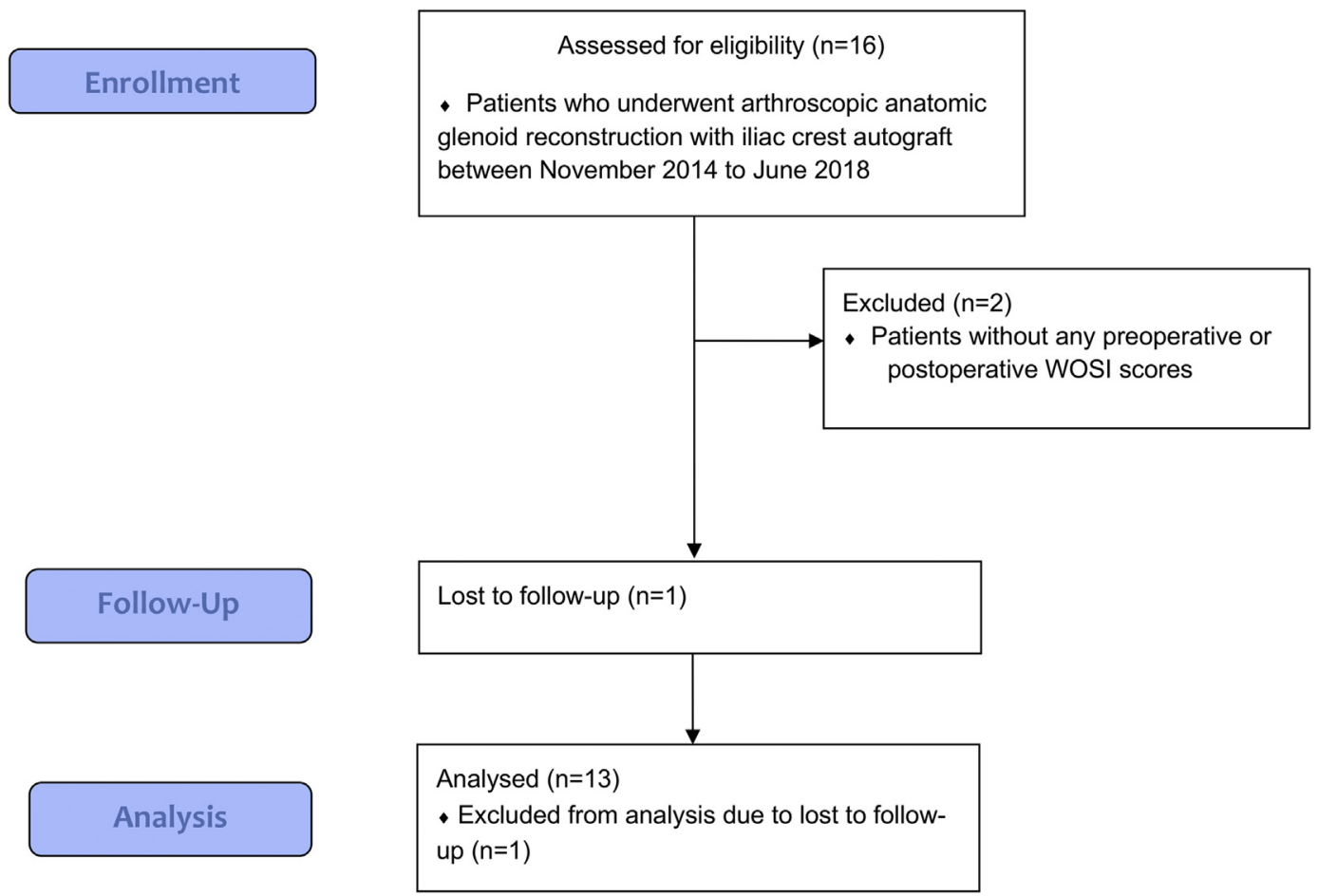

Fig 1. CONSORT (Consolidated Standards of Reporting Trials) flow chart of patient selections, including the number of patients initially included due to the inclusion criterion $(n=16)$, the number of the patients excluded due to missing patient-reported outcomes $(n=2)$, the number of patients lost to follow-up $(n=1)$, and also the number of the patients finally included in the analysis $(\mathrm{n}=13)$.

reported iliac crest donor site pain; however, no patients required further surgery for any complication at the iliac crest donor sites.

\section{Clinical Outcomes}

Short-term clinical outcomes were found to be favorable. None of the patients experienced dislocation during follow-up nor presented with a positive shoulder apprehension test during clinical examination. There was a significant improvement from preoperative

Table 1. Study Population Demographics and Clinical Characteristics

\begin{tabular}{lc}
\hline \multicolumn{1}{c}{ Preoperative Findings } & $\mathrm{N}(\%)$ \\
\hline Number of patients & 13 \\
Follow-up, y, mean \pm SD & $1.4 \pm 1.3$ \\
Age at surgery, y, mean \pm SD & $30.6 \pm 14.2(16.3-70.5)$ \\
Sex & $8(61.5 \%)$ \\
$\quad$ Male & $5(38.5 \%)$ \\
Female & $7(53.8 \%)$ \\
Side & $6(46.2 \%)$ \\
$\quad$ Right & $7.33 \pm 2.85(20 \%-45 \%)$ \\
Left & $10(76.9 \%)$ \\
Glenoid bone loss, mm; AP, mean \pm SD & $3(23.1 \%)$ \\
Indications & \\
$\quad$ Bone loss & \\
Bone loss and previous failed surgery & \\
\hline AP, anteroposterior; SD, standard deviation. &
\end{tabular}

$70.4 \pm 23.3$ to postoperative $37.9 \pm 20.6$ (lower score is better) $(P<.001$, Hedges' $\mathrm{g}=1.48)$ (Table 2$)$. There were $91 \%$ of patients who met the minimal clinically important differences of the WOSI scores.

\section{Radiographic Outcomes}

Postoperative CT scans were performed to assess bone healing and progress of rehabilitation on 12 patients $(92.3 \%)$, with a mean of 11.0 months between the surgery and CT scan (Table 3). Eleven of 12 patients $(91.7 \%)$ displayed complete graft union. One patient $(8.3 \%)$ had partial nonunion, as there was nonunion of the proximal graft but healing of the inferior graft. The mean preoperative anterior-to-posterior dimension of the glenoid was $22.0 \pm 3.1 \mathrm{~mm}$. Postoperatively the mean anterior-to-posterior dimension of the glenoid with graft was $29.3 \pm 1.8 \mathrm{~mm}$. Graft resorption was noted in 11 patients $(91.7 \%)$. Of the 11 patients with

Table 2. Pre- and Postoperative Western Ontario Shoulder Instability Index (WOSI) Scores

\begin{tabular}{ll}
\hline \multicolumn{1}{c}{ Outcome } & Mean \pm SD \\
\hline Preoperative WOSI Scores & $70.4 \pm 23.3$ \\
Postoperative WOSI Scores & $37.9 \pm 20.6$ \\
$\Delta$ WOSI & $35.0 \pm 20.2$ \\
\hline SD, standard deviation
\end{tabular}


Table 3. Postoperative CT Findings $(\mathrm{N}=12)$

\begin{tabular}{llc}
\hline \multicolumn{1}{c}{ CT Assessments } & $\mathrm{N}$ & Mean \pm SD \\
\hline Time between surgery and CT, mo & 12 & $11.0 \pm 13.79$ \\
AP dimension without graft, mm & 12 & $22.0 \pm 3.1$ \\
AP dimension with graft, mm & 12 & $29.3 \pm 1.8$ \\
Graft size, mm & 12 & $7.3 \pm 3.0$
\end{tabular}

AP, anteroposterior; CT, computed tomography; SD, standard deviation.

resorption, $6(54.5 \%)$ had $<25 \%$ resorption, $4(36.4 \%)$ had $25-50 \%$ resorption, and 1 patient $(9.1 \%)$ had $>50 \%$ resorption. In the 2 patient with $>50 \%$ resorption, there remained an autograft sagittal dimension of $3.6 \mathrm{~mm}$. There are no statistically significant differences among the patients with different graft resorptions in terms of anteroposterior dimensions with and without graft, and graft sizes (Table 4). All patients had the ideal vertical graft position (i.e., 3-5 o'clock) and 11 patients' graft positionings (92\%) were flush with the glenoid (i.e., ML step $=0$ ). There was 1 patient whose graft had $1.9 \mathrm{~mm}$ medial step that is also within the acceptable range.

\section{Discussion}

The AAGR using an autologous iliac crest bone graft showed good results regarding both the safety profile and short-term clinicoradiographic outcomes, especially when considering that our study population had a high risk for failure and recurrence of shoulder instability. As the average anterior-to-posterior dimension of the glenoid has been reported to range from 23 to 25 $\mathrm{mm},{ }^{20-22}$ our patient population had a mean glenoid bone loss of approximately 30\%. No intraoperative complications were observed, including neurovascular injury or major bleeding. There were no instances of infection postoperatively. No patients experienced subluxation during follow-up nor presented with a positive shoulder apprehension test during clinical examination. Also, WOSI index scores assessing the impact of injury on shoulder function within domains of physical function, recreation, lifestyle and emotional well-being improved significantly postoperatively.

The excellent safety profile for AAGR using autologous iliac crest bone graft can likely be attributed to keeping the subscapularis muscle and conjoint tendons intact. These structures can be visualized and protected through the establishment of the Halifax portal via an inside-out technique, where the switching stick is passed through the rotator interval superior to the subscapularis and lateral to the conjoint tendon. In fact, the Halifax portal is on average a minimum of $4.5 \mathrm{~cm}$ away from the musculocutaneous and axillary nerves since they are shielded by the intact subscapularis muscle and conjoint tendon. ${ }^{16}$ In addition, the risk of neurovascular injury is minimized with this arthroscopic technique, as it does not require the use of significant tissue or periarticular retraction necessary for open reconstruction techniques.

Conversely, some of the most prominent concerns regarding the safety profile of the Latarjet procedure are the injury to the musculocutaneous and axillary nerves. Delaney et al. ${ }^{23}$ used neuromonitoring to find that 7 of 34 patients $(20.6 \%)$ suffered from axillary nerve deficit following a Latarjet procedure. Shah et al. ${ }^{24}$ similarly recorded 5 of 50 patients $(10 \%)$ to have neurologic injury after Latarjet reconstruction, including 2 instances of injury to the musculocutaneous nerve, 2 to the axillary nerve, and 1 involving injury to the radial nerve. Perhaps most notably, a systematic review of 45 studies by Griesser et al. ${ }^{25}$ found the total complication rate to be $30 \%$, including neurovascular injury, redislocation, hematoma, and graft fracture. AAGR using iliac crest autograft certainly demonstrates a favorable safety profile in comparison, although we did not use the same method of determining nerve-related complications.

In comparison with the Latarjet procedure, the technique described in this study also benefits from being an anatomic reconstruction, as there is no requirement for repositioning the conjoint tendon or splitting the subscapularis muscle for graft placement. The original anatomy of the shoulder is conserved, as are its biomechanics. Other anatomic bony reconstruction techniques using distal tibial allograft have been shown to offer a comparable safety profile and clinical outcomes to iliac crest bone graft, ${ }^{26,27}$ although with increased monetary cost and small risk of disease transmission or immune response provocation due to use of an allograft. In contrast, sourcing the graft from the patient's iliac crest is less expensive and inherently

Table 4. Comparison of Patients With Different Graft Resorptions in Terms of AP Dimensions With and Without Graft, and Graft Sizes

\begin{tabular}{lcccc}
\hline \multicolumn{1}{c}{ Graft Resorption } & $\mathrm{N}$ & AP Dimension Without Graft, mm & AP Dimension With Graft, mm & Graft Size, mm \\
\hline No resorption & 1 & 17.6 & 28.8 & 11.2 \\
$<25 \%$ resorption & 6 & $22.9 \pm 3.7$ & $30.6 \pm 1.1$ & $7.7 \pm 3.5$ \\
Resorption between $25 \%$ and $50 \%$ & 4 & $21.3 \pm 1.7$ & $28.1 \pm 1.9$ & 27.3 \\
$>50 \%$ resorption & 1 & 23.7 & .084 & 3.6 \\
$P$ value (alpha $=0.05)$ & & .433 & .361 \\
\hline
\end{tabular}

AP, anteroposterior; CT, computed tomography; SD, standard deviation. 
allows for excellent graft availability, at the cost of risk of donor-site morbidity. Although the iliac crest is a common donor site due to low morbidity, ${ }^{28} 13 \%$ to $20 \%$ of patients may experience chronic pain. ${ }^{29,30}$ This should be taken into consideration, yet may be offset against the significant improvement in quality of life experienced by these patients, as reflected by the improvement in WOSI scores.

In this study 11 of 12 patients $(91.7 \%)$ who had postoperative CT imaging experienced some degree of bone graft resorption as seen on CT imaging. This is in line with the findings of Hoffelner et al., ${ }^{31}$ who reported that $100 \%$ of 11 patients experienced bone resorption after having undergone revision shoulder arthroplasty with bone graft. Another retrospective review of 11 patients with bone grafts to correct for severe glenoid defects by Scalise and Iannotti ${ }^{32}$ also saw $100 \%$ of subjects demonstrate resorption. There are other studies that report lower rates of allograft and autograft reabsorption in the context of significant glenoid bone loss ${ }^{26,33-35}$; however, it is important to note that even though most patients in our study population experienced resorption, there were no instances of graft failure or clinical instability. Even in the one patient with $>50 \%$ resorption, there remained an autograft sagittal dimension of $3.55 \mathrm{~mm}$, enough to maintain clinical stability of the shoulder. Overall, the occurrence of graft reabsorption in the majority of patients in our study could be explained by Wolff's law, where if a placed graft caused the glenoid to be larger than its physiological size, it would undergo physiological reabsorption to remodel to native bony anatomy. ${ }^{36}$

This study investigated the clinical outcomes of an arthroscopic iliac crest autograft and also included the use of both clinical and radiological data to aid in forming more comprehensive comparisons of preoperative and postoperative measurements. Furthermore, the data were collected from 2 different centers with 2 different surgeons.

\section{Limitations}

The main limitations of this study are its small sample size and retrospective nature, as the reviewing of patient charts to assess for intraoperative and postoperative complications could be a potential source for error due to the limited information available in these charts. One must also consider that we only have follow-up beyond 2 years postoperatively in 3 of 14 patients $(21.4 \%)$. Recurrent instability is the most common complication following a procedure involving anterior stabilizing and is more likely to occur later on. Further investigations are required to evaluate the longevity of these positive health outcomes.

\section{Conclusions}

Arthroscopic treatment of shoulder instability with bone loss via autologous iliac crest bone graft is shown to be a safe operative procedure that results in favourable short-term clinical and radiological outcomes, with a significant improvement in WOSI scores and high rates of graft union. Although graft resorption was seen in most patients who had postoperative CT imaging, there were no instances of clinical graft failure.

\section{Acknowledgments}

We thank Sara Sparavalo and Ryland Murphy for assistance in study protocol preparation, obtaining research ethics board approval, and data collection and management and Jie Ma for assistance in obtaining research ethics board approval, data management and analysis, and paper submission.

\section{References}

1. Uhorchak JM, Arciero RA, Huggard D, Taylor DC. Recurrent shoulder instability after open reconstruction in athletes involved in collision and contact sports. Am J Sports Med 2000;28:794-799.

2. Taylor DC, Arciero RA. Pathologic changes associated with shoulder dislocations: Arthroscopic and physical examination findings in first-time, traumatic anterior dislocations. Am J Sports Med 1997;25:306-311.

3. Gottschalk IV LJ, Bois AJ, Shelby MA, Miniaci A, Jones MH. Mean glenoid defect size and location associated with anterior shoulder instability: A systematic review. Orthop J Sports Med 2017;5:2325967116676269.

4. Burkhart SS, De Beer JF. Traumatic glenohumeral bone defects and their relationship to failure of arthroscopic Bankart repairs: Significance of the inverted-pear glenoid and the humeral engaging Hill-Sachs lesion. Arthroscopy 2000;16:677-694.

5. Balg F, Boileau P. The instability severity index score. J Bone Joint Surg Br 2007;89-B:1470-1477.

6. Boileau P, Villalba M, Héry J-Y, Balg F, Ahrens P, Neyton L. Risk factors for recurrence of shoulder instability after arthroscopic Bankart repair. J Bone Joint Surg 2006;88:1755-1763.

7. Allain J, Goutallier D, Glorion C. Long-term results of the Latarjet procedure for the treatment of anterior instability of the shoulder. J Bone Joint Surg Am 1998;80:841-852.

8. Hovelius L, Sandstorm B, Rosmark D, Saebo M, Sundgren K, Malmqvist B. Long-term results with the Bankart and Bristow Latarjet procedures: Recurrent shoulder instability and arthropathy. J Shoulder Elbow Surg 2001;10:445-452.

9. Longo UG, Loppini M, Rizzello G, Ciuffreda M, Maffulli N, Denaro V. Latarjet, Bristow, and Eden-Hybinette procedures for anterior shoulder dislocation: Systematic review and quantitative synthesis of the literature. Arthroscopy 2014;30:1184-1211.

10. Warner JJP, Gill TJ, O'hollerhan JD, Pathare N, Millett PJ. Anatomical glenoid reconstruction for recurrent anterior glenohumeral instability with glenoid deficiency using an 
autogenous tricortical iliac crest bone graft. Am J Sports Med 2006;34:205-212.

11. Gupta A, Delaney R, Petkin K, Lafosse L. Complications of the Latarjet procedure. Curr Rev Musculoskelet Med 2015;8:59-66.

12. Lafosse L, Boyle S. Arthroscopic Latarjet procedure. J Shoulder Elbow Surg 2010;19:2-12 (suppl 2).

13. Lewington MR, Urquhart N, Wong IH. Lateral decubitus allarthroscopic Latarjet procedure for treatment of shoulder instability. Arthrosc Tech 2015;4:e207-e213.

14. Kraus N, Amphansap T, Gerhardt C, Scheibel M. Arthroscopic anatomic glenoid reconstruction using an autologous iliac crest bone grafting technique. J Shoulder Elbow Surg 2014;23:1700-1708.

15. Wong IH, Urquhart N. Arthroscopic anatomic glenoid reconstruction without subscapularis split. Arthrosc Tech 2015:4:e449-e456.

16. Moga I, Konstantinidis G, Wong IH. The safety of a far medial arthroscopic portal for anatomic glenoid reconstruction: A cadaveric study. Orthop J Sports Med 2018;6: 2325967118795404

17. John R, Wong I. Arthroscopic revision with autologous iliac crest bone graft for failed anatomic glenoid reconstruction using distal tibia allograft. Arthrosc Tech 2019;8:e1333-el338.

18. Kirkley A, Griffin S, McLintock H, Ng L. The development and evaluation of a disease-specific quality of life measurement tool for shoulder instability. The Western Ontario Shoulder Instability Index (WOSI). Am J Sports Med 1998;26:764-772.

19. Kirkley A, Griffin S, Dainty K. Scoring systems for the functional assessment of the shoulder. Arthroscopy 2003;19:1109-1120.

20. Iannotti JP, Gabriel JP, Schneck SL, Evans BG, Misra S. The normal glenohumeral relationships. An anatomical study of one hundred and forty shoulders. J Bone Joint Surg Am 1992;74:491-500.

21. Mamatha T, Pai SR, Murlimanju BV, Kalthur SG, Pai MM, Kumar B. Morphometry of glenoid cavity. Online J Health Allied Sci $2011 ; 10: 7$.

22. Piponov HI, Savin D, Shah N, et al. Glenoid version and size: Does gender, ethnicity, or body size play a role? Int Orthop 2016:40:2347-2353.

23. Delaney RA, Freehill MT, Janfaza DR, Vlassakov KV, Higgins LD, Warner JJP. Neer Award Paper: Neuromonitoring the Latarjet procedure. J Shoulder Elbow Surg 2014;23:1473-1480.

24. Shah AA, Butler RB, Romanowski J, Goel D, Karadagli D, Warner JJ. Short-term complications of the Latarjet procedure. J Bone Joint Surg Am 2012;94:495-501.
25. Griesser MJ, Harris JD, McCoy BJ, et al. Complications and re-operations after Bristow-Latarjet shoulder stabilization: A systematic review. J Shoulder Elbow Surg 2013;22:286-292.

26. Amar E, Konstantinidis G, Coady C, Wong IH. Arthroscopic treatment of shoulder instability with glenoid bone loss using distal tibial allograft augmentation: Safety profile and short-term radiological outcomes. Orthop J Sports Med 2018;6:2325967118774507.

27. Wong I, John R, Ma J, Coady CM. Arthroscopic anatomic glenoid reconstruction using distal tibial allograft for recurrent anterior shoulder instability: Clinical and radiographic outcomes. Am J Sports Med 2020;48: 3316-3321.

28. Zhang J, Wei Y, Gong Y, et al. Reconstruction of iliac crest defect after autogenous harvest with bone cement and screws reduces donor site pain. BMC Musculoskelet Disord 2018;19:237.

29. Armaghani SJ, Even JL, Zern EK, Braly BA, Kang JD, Devin CJ. The evaluation of donor site pain after harvest of Tricortical anterior iliac crest bone graft for spinal surgery: A prospective study. Spine (Phila Pa 1976) 2016;41: E191-E196.

30. Kim DH, Rhim R, Li L, et al. Prospective study of iliac crest bone graft harvest site pain and morbidity. Spine J 2009;9: 886-892.

31. Hoffelner T, Moroder P, Auffarth A, Tauber M, Resch H. Outcomes after shoulder arthroplasty revision with glenoid reconstruction and bone grafting. Int Orthop 2014;38: 775-782.

32. Scalise JJ, Iannotti JP. Bone grafting severe glenoid defects in revision shoulder arthroplasty. Clin Orthop Relat Res 2008;466:139-145.

33. Iannotti JP, Frangiamore SJ. Fate of large structural allograft for treatment of severe uncontained glenoid bone deficiency. J Shoulder Elbow Surg 2012;21: 765-771.

34. Phipatanakul WP, Norris TR. Treatment of glenoid loosening and bone loss due to osteolysis with glenoid bone grafting. J Shoulder Elbow Surg 2006;15:84-87.

35. Ueda Y, Sugaya H, Takahashi N, et al. Arthroscopic iliac bone grafting for traumatic anterior shoulder instability with significant glenoid bone loss yields low recurrence and good outcome at a minimum of five-year follow-up. Arthroscopy 2021;37:2399-2408.

36. Frost HM. Wolff's law and bone's structural adaptations to mechanical usage: An overview for clinicians. Angle Orthod 1994;64:175-188. 